INPLASY

PROTOCOL

To cite: Zhu et al. Guizhi Fuling Combined with Antibiotic Therapy for Pelvic Inflammatory Disease: A Systematic Review and MetaAnalysis. Inplasy protocol 2020100035. doi: 10.37766/inplasy2020.10.0035

Received: 10 October 2020

Published: 10 October 2020

Corresponding author: Rui Zhu

zr330194311@outlook.com

Author Affiliation:

Department of Traditional

Chinese Medicine, Medical

College of Qinghai University

Support: 2020-ZJ-949Q.

Review Stage at time of this submission: The review has not yet started.

Conflicts of interest:

The authors declare that they have no conflicts of interest.

\section{Guizhi Fuling Combined with Antibiotic Therapy for Pelvic Inflammatory Disease: A Systematic Review and Meta-Analysis}

Zhu, R' Li, M2; Ma, X³.

Review question / Objective: The aim of this meta-analysis of randomized controlled trials is to evaluate the efficacy and safety of Guizhi Fuling (GZFL) combined with antibiotics regimens and antibiotics alone in the treatment of Pelvic Inflammatory Disease.

Condition being studied: PID is a spectrum of inflammatory disorders of the upper female genital tract. The age is mainly concentrated from 25 to 35 , and more than $1 \%$ of sexually mature females are estimated to be suffering from PID. Reproductive tract infection, especially N. gonorrhoeae and C. trachomatis, and uterine cavity operation are the most common causes of PID. However, due to the inappropriate and irrational use of antibiotics, there are problems of drugresistant bacteria, reduced drug sensitivity, and some side effects. Traditional Chinese medicine (TCM) has a longstanding history, with exact efficacy and obvious advantages in treating gynecological diseases. Evidence suggests that Chinese patent medicine combined with antibiotics in the treatment of PID has a remarkable curative effect, improves the clinical symptoms effectively, and reduces the relapse rate notably.

INPLASY registration number: This protocol was registered with the International Platform of Registered Systematic Review and Meta-Analysis Protocols (INPLASY) on 10 October 2020 and was last updated on 10 October 2020 (registration number INPLASY2020100035).

\section{INTRODUCTION}

Review question / Objective: The aim of this meta-analysis of randomized controlled trials is to evaluate the efficacy and safety of Guizhi Fuling (GZFL) combined with antibiotics regimens and antibiotics alone in the treatment of Pelvic Inflammatory Disease.

Condition being studied: PID is a spectrum of inflammatory disorders of the upper female genital tract. The age is mainly concentrated from 25 to 35 , and more than 
$1 \%$ of sexually mature females are estimated to be suffering from PID.Reproductive tract infection, especially $\mathrm{N}$. gonorrhoeae and C. trachomatis, and uterine cavity operation are the most common causes of PID. However, due to the inappropriate and irrational use of antibiotics, there are problems of drugresistant bacteria, reduced drug sensitivity, and some side effects. Traditional Chinese medicine (TCM) has a long-standing history, with exact efficacy and obvious advantages in treating gynecological diseases. Evidence suggests that Chinese patent medicine combined with antibiotics in the treatment of PID has a remarkable curative effect, improves the clinical symptoms effectively, and reduces the relapse rate notably.

\section{METHODS}

Participant or population: Patients of childbearing age diagnosed with PID by clinical symptoms or auxiliary examinations based on the criteria of guidelines and teaching materials. Patients who are systemically unwell, with presence of a tubo-ovarian abscess, who are pregnant, undergoing surgery, or after salpingography were excluded.

Intervention: Guizhi Fuling combined with antibiotic therapy were the main intervention.

\section{Comparator: Use antibiotics alone.}

Study designs to be included: Data from RCTs and quasi-randomized trials were sought electronically. The RCTs comparing GZFL combined with antibiotic therapy for PID were included.

Eligibility criteria: This review is confined to RCTs comparing GuizhiFuling(GZFL) combined with antibiotics regimens with antibiotics regimens alone, which contained other drugs, no treatment, placebo, diet and exercise therapy, acupuncture, and so forth. It is deemed a randomized study if the trial stated the "randomization" phrase, and the blinding is not restricted. Besides, Chinese and
English publications are the limitation of language. The animal mechanism studies, case reports, self-pre-and postcontrol, or non-RCTs are excluded.

Information sources: A literature search will be conducted up to October 2020 . We will electronically search the randomized controlled trials in the following databases: including the Chinese Biomedical Literature Database (CBM), MEDLINE, PubMed, the Cochrane Library, Excerpta Medica (EMBASE), the China National Knowledge Infrastructure Database (CNKI), the Wanfang database and the Chinese Scientific Journal Database (VIP database).

Main outcome(s): Primary outcomes consisted of the rate of improvement and adverse events.

Quality assessment / Risk of bias analysis: Two reviewers will independently evaluate the risk of bias for the included studies using the Cochrane Collaboration's tool for evaluating the risk of bias, consensus, or consulting with the third author if necessary. The tool supports the consideration of sequence generation, allocation concealment, blinding, incomplete outcome data, selective outcome reporting, and other sources of bias. We will assess the risk of bias in each source as low risk of bias, high risk of bias, and unclear risk of bias and contacte the study's authors to request for missing information by using open-ended questions if necessary. If more than 10 trials are included, funnel plots will be used to assess reporting biases. If funnel plot asymmetry is detected, the reasons will be analyzed.

Strategy of data synthesis: We will use Revman software provided by the Cochrane collaboration to analyze the data. Binary outcomes will be summarized using risk ratio (RR) with $95 \%$ confidence interval (CI) for relative effect. Continuous outcomes will be summarized by using weighted mean difference (WMD) with $95 \%$ Cl. We will use random-effect model (REM) for meta-analysis in this review according 
to research recommendations. Statistical heterogeneity will be assessed by $X^{2}$ and $I^{2}$ statistical tests. Where $p$ value $\geq 0.1$ and $I^{2}$ $\leq 50 \%$, there is no obvious statistical heterogeneity among the studies. On the contrary, where $p$ value $50 \%$ indicates a considerable heterogeneity. Meta-analysis will be performed when the statistical heterogeneity is acceptable ( $p$ value $\geq 0.1$ and $I^{2} \leq 50 \%$ ), otherwise, subgroup analysis will be applied to explore with careful consideration.

Subgroup analysis: A subgroup analysis will be performed according to control intervention and different outcomes.

Sensibility analysis: In order to assure the robustness of our results, We will conduct sensitivity analysis to eliminate the impact of low-quality studies, with the premise of significant heterogeneity still exists right after validation of inputted data and subgroup analysis. We will contrast the results of these two meta-analyses and decide whether to exclude low-quality researches based on impact on pooled effective size, sample size and strength of evidence. Nevertheless, if all included studies are at high risk of bias, we will not conduct sensitivity analysis.

Country(ies) involved: China.

Keywords: Guizhi Fuling; Pelvic inflammation disease; Herbal medicine; Systematic review; Meta-Analysis.

Contributions of each author:

Author 1 - Rui Zhu - The author drafted and improved the manuscript. The author will contribute to the development of the selection criteria, data synthesis, and the risk of bias assessment strategy.

Author 2 - Mingle Li - The author drafted and improved the manuscript. This author will contribute to the the development of the selection criteria, data synthesis and sensitivity analysis.

Author 3 - Xueman Ma - Two reviewers will independently evaluate the risk of bias for the included studies using the Cochrane Collaboration's tool for evaluating the risk of bias, consensus, or consulting with the corresponding author if necessary. This author is the guarantor for the veracity and accuracy of the protocol. 